\section{Cureus}

\title{
Post-Traumatic, Drug-Resistant Epilepsy and Review of Seizure Control Outcomes from Blinded, Randomized Controlled Trials of Brain Stimulation Treatments for Drug-Resistant Epilepsy
}

\author{
Michael Larkin $^{1}$, R. Michael Meyer ${ }^{1}$, Nicholas S. Szuflita ${ }^{1}$, Meryl A. Severson ${ }^{2}$, Zachary T. Levine ${ }^{3}$
}

1. School of Medicine, Uniformed Services University of the Health Sciences 2. Department of Neurosurgery, Walter Reed National Military Medical Center/Uniformed Services University of Health Sciences 3. Department of Neurosurgery, Holy Cross Hospital

$\square$ Corresponding author: Michael Larkin, michael.larkin@usuhs.edu Disclosures can be found in Additional Information at the end of the article

\section{Abstract}

Background: Many post-traumatic epilepsy (PTE) patients become resistant to medications. Nervous stimulation as a treatment for drug-resistant epilepsy (DRE) is an active area of clinical investigation.

Objective: To summarize methods, reported seizure control outcome measures, and adverse events from blinded, randomized control trials (RCTs) for selected invasive brain stimulation (IBS) and non-invasive brain stimulation (NIBS) treatment options in patients with DRE.

Methods: PubMed was searched for articles from 1995-2014, using search terms related to the topics of interest. Available relevant articles reporting the outcomes of interest were identified and data was extracted. Articles in the reference lists of relevant articles and clinicaltrials.gov were also referenced.

Results: Eleven articles were analyzed with a total of 795 patients identified. Studies showed that select nervous stimulation treatments significantly reduced seizure frequency in patients with DRE.

Categories: Neurology, Neurosurgery

Keywords: post-traumatic epilepsy, vagus nerve stimulation, deep brain stimulation, anterior nucleus thalami, responsive cortical neurostimulation, transcranial direct current stimulation, repetitive transcranial magnetic stimulation, drug-resistant epilepsy

Received 06/26/2016 Review began 07/06/2016 Review ended 08/07/2016 Published 08/22/2016

C Copyright 2016

Larkin et al. This is an open access article distributed under the terms of the Creative Commons Attribution License CC-BY 3.0., which permits unrestricted use distribution, and reproduction in any medium, provided the original author and source are credited.

\section{Introduction And Background}

Epilepsy affects more than 65 million of the worldwide population and 2.2 million people in the United States [1]. In the general population, up to $20 \%$ of acquired symptomatic epilepsy results from traumatic brain injury (TBI) [2]. The risk of developing post-traumatic epilepsy (PTE) has been described as being up to a 30-50\% after a TBI; a 30 times greater risk of epilepsy when compared to the general population [3-4]. Shortly after the TBI, there is a cascade of events which include contusions, cytotoxic edema, axonal shearing, inflammatory responses, free-radical generation, mitochondrial dysfunction, neurotransmitter release, and angiogenesis [5]. These changes cause an imbalance between excitatory and inhibitory processes and lead to an increased risk for the formation of spontaneous epileptic foci due to excessive excitatory stimulation [6]. Post-traumatic seizures can occur as soon as one week after injury or up to ten years or longer post-TBI [7]. For patients who have a single late seizure (>7 days TBI), more than $80 \%$ will have their second seizure within two years and will then have up to a $90 \%$ chance of progressing to PTE [78]. Antiepileptic drugs (AEDs) have only been demonstrated to prevent the occurrence of early seizure onset but do not prevent the late development of PTE, and their use should be discontinued after seven days if no seizures occur [9]. Currently, there are no known effective therapies to prevent PTE and no way for clinicians to accurately predict who will develop PTE. A majority of these patients will also develop
How to cite this article

Larkin M, Meyer R, Szuflita N S, et al. (August 22, 2016) Post-Traumatic, Drug-Resistant Epilepsy and Review of Seizure Control Outcomes from Blinded, Randomized Controlled Trials of Brain Stimulation Treatments for Drug-Resistant Epilepsy. Cureus 8(8): e744. DOI 10.7759/cureus.744 
drug-resistant epilepsy (DRE)--defined as a failure of an adequate trial of $>2$ tolerated, appropriately chosen, and used AED regimens (monotherapy or combination) to achieve seizure cessation [10-11].

In general, AEDs result in seizure control in two-thirds of epilepsy patients, and another 7-8\% of patients can be cured with surgery [5, 12]. PTE is complicated by diffuse epileptogenicity, which makes it difficult to locate areas for resection on the basis of sensor-space EEG analysis [13]. However, localization has been improved with the use of "inverse" EEG techniques that have only recently become available and are out of the scope of this review [5]. PTE is further complicated by frequently present, wide-spread encephalomalacia. The surgical removal of these damaged areas can be effective, but the inability to consistently localize seizure foci in PTE patients continues to be a barrier for complete surgical resection. Thus, there continues to be an unmet need for additional antiepileptic treatment options, as some PTE patients will not be operative candidates, and up to one-third will remain drug-resistant.

Nervous stimulation as a treatment for DRE is an active area of clinical investigation. This article will summarize the methods, reported seizure control outcome measures, and adverse events from blinded, randomized control trials (RCTs) for several invasive brain stimulation (IBS) and non-invasive brain stimulation (NIBS) therapies that have been applied to the treatment of DRE, including vagal nerve stimulation (VNS), deep brain stimulation of the anterior thalamus (DBS-ANT), responsive cortical neurostimulation (RNS), transcranial direct current stimulation (tDCS), and repetitive transcranial magnetic stimulation (rTMS). Currently, the IBS methods are the only therapies approved for clinical use. The purpose of this review is to highlight the use of the described nervous stimulation therapies for the treatment of DRE and to encourage further attention and funding be given to advance the clinical care and patient outcomes for patients presenting with post-traumatic, drug-resistant epilepsy (PTDRE). To the authors' knowledge, no such studies have been conducted.

\section{Review}

\section{Materials and methods}

\section{Literature Search}

Studies were identified via a PubMed search for articles from 1995-2014, using search terms related to the treatment method: VNS, DBS, RNS, tDCS, and rTMS; and in conjunction with the keywords "seizure or epilepsy", "frequency", and "decrease or reduction". The reference lists of these articles were then examined for additional resources that fit the selection criteria. An ongoing clinical trial from clinicaltrials.gov was also included in the review. One article was discovered incidentally during the fulltext review of articles.

\section{Selection Criteria}

The following criteria were utilized for article selection: (1) articles in English, (2) human trials, (3) > 12 years of age, (4) controlled trials, (5) blinded, and (6) randomized. Articles selected for full-text review were further excluded if our detailed review revealed that the above criteria were not met.

\section{Data Extraction}

The primary author (MBL) identified relevant articles reporting the outcomes of interest. Two authors (MBL, RMM) extracted relevant data independently. Each author's data was cross-reviewed by the other, and any discrepancies, if necessary, were resolved by a third author (MAS). Standardized tables were utilized to extract the following variables: (1) seizure frequency reduction, (2) seizure frequency risk reduction, and (3) percent responders. The primary author collected the reported demographic characteristics, study design, stimulation parameters, and adverse events.

\section{Results}

In total, 370 articles were identified. After applying the selection criteria, 11 relevant articles were identified with a total of total of 795 patients (Figure 1). In general, studies showed that select nervous stimulation modalities significantly reduced seizure frequency in select DRE patients. 


\section{Cureus}

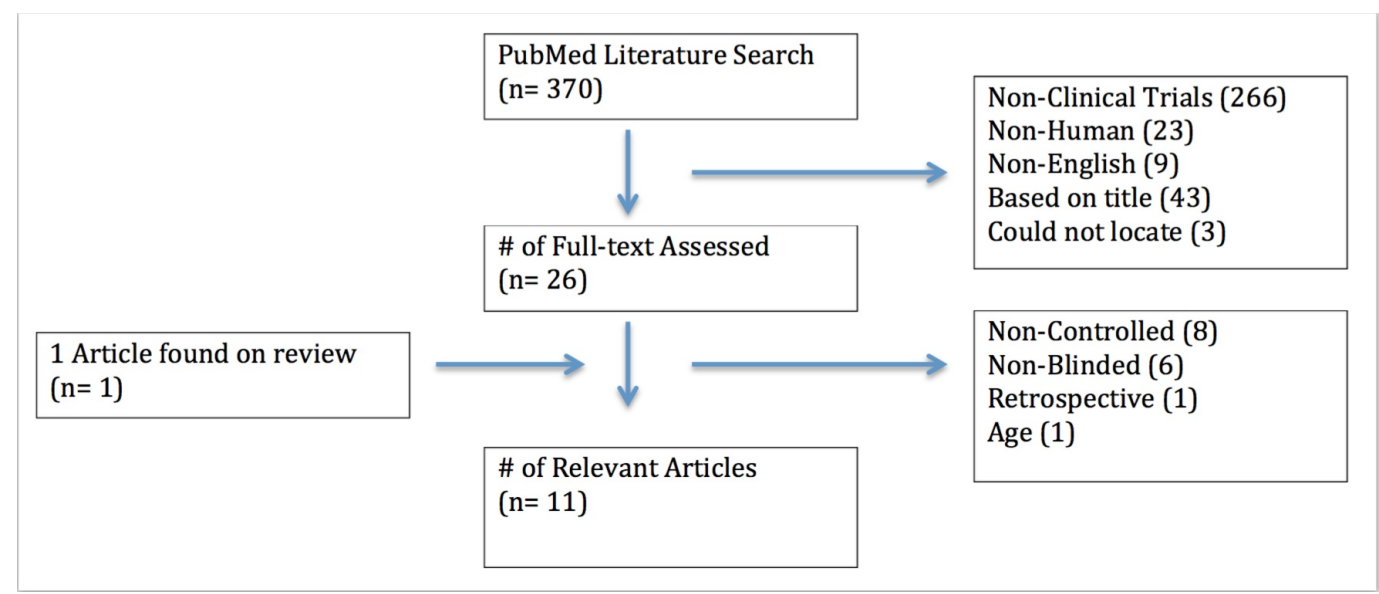

FIGURE 1: Search Flow Diagram

\section{Discussion}

Vagus Nerve Stimulation

In 1997, the Food and Drug Administration (FDA) approved VNS for adjunctive therapy for DRE in patients greater than twelve years old [14]. VNS devices (Figure 2) generally consist of an implantable pulse generator that is placed in the left sub-clavicular fossa with electrodes that are wrapped around the left vagus nerve distal to the take-off of the cardiac branch [15]. Typically, the right vagus nerve is avoided due to the increased potential for negative effects because of its greater role in cardiac function [16]. The exact mechanism for reduced seizure activity in VNS remains unknown. The majority of vagus nerve fibers are afferent, and the antiepileptic properties of VNS are thought to be related to these afferent effects on the brainstem reticular activating system, the central autonomic network, the limbic system, and the diffuse noradrenergic projection system; all of which have connections to numerous forebrain structures involving multiple neurotransmitters (norepinephrine and GABA) [17-20]. 


\section{Cureus}

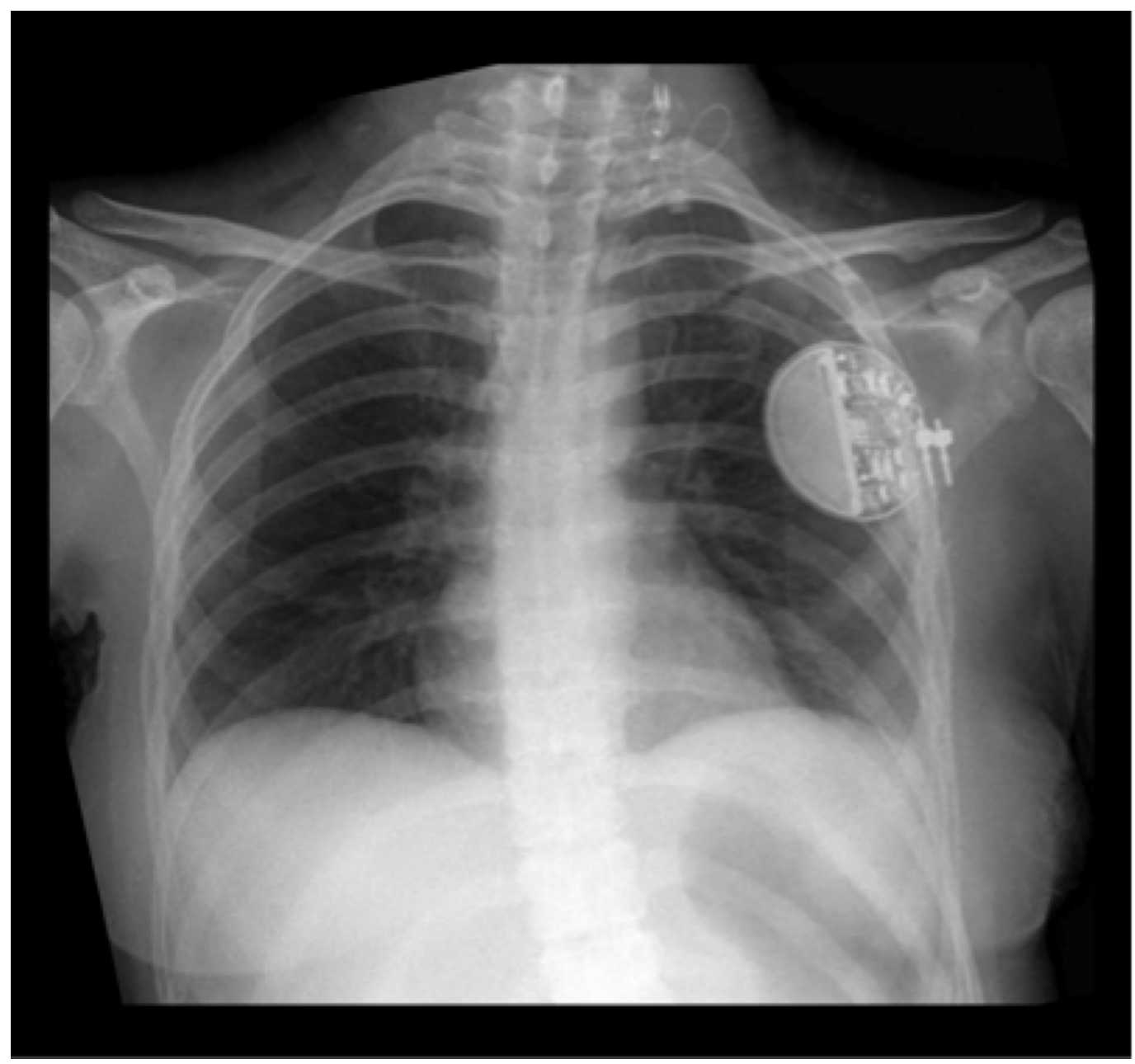

FIGURE 2: Vagal Nerve Stimulator

Case courtesy of Dr. Jayanth Keshavamurthy; Radiopaedia.org, rID: 26836

Three, double-blinded, randomized controlled trials have been published for the use of VNS in the treatment of DRE [15, 21-22]. Significant changes in seizure frequency from baseline in the high stimulation groups of the E03, E05, and Amar, et al. studies when compared to the low stimulation groups as well as participant demographics and stimulation parameters are summarized in Table 1 . The rates of responders in the treatment groups (defined as those participants with $\mathrm{a} \geqslant 50 \%$ decrease in seizure frequency) were $31 \%, 23.4 \%$, and $57 \%$, respectively. 


\section{Cureus}

\begin{tabular}{|c|c|c|c|c|c|c|c|c|c|c|c|c|c|}
\hline Study & $\begin{array}{l}\text { \# } \\
\text { Pts }\end{array}$ & $\begin{array}{l}\text { Age } \\
\text { (yrs) }\end{array}$ & \#AEDs & $\begin{array}{l}\text { Stimulation } \\
\text { Group }\end{array}$ & $\begin{array}{l}\text { Current } \\
\text { (mA) }\end{array}$ & $\begin{array}{l}\text { Pulse } \\
\text { Time } \\
\text { ( } \mu \mathrm{sec})\end{array}$ & $\begin{array}{l}\text { Freq } \\
(\mathrm{Hz})\end{array}$ & $\begin{array}{l}\text { ON Time } \\
\text { (sec) }\end{array}$ & $\begin{array}{l}\text { OFF Time } \\
(\min )\end{array}$ & $\begin{array}{l}\text { Duration } \\
\text { of BEP } \\
\text { (weeks) }\end{array}$ & $\begin{array}{l}\% \\
\text { SFR* }\end{array}$ & SFRR* & $\begin{array}{l}\% \\
\text { Resp* }\end{array}$ \\
\hline \multicolumn{14}{|l|}{ VNS } \\
\hline \multirow{2}{*}{$\begin{array}{l}\text { VNSSG } 1995 \text { (E03) } \\
{[15]}\end{array}$} & \multirow{2}{*}{114} & \multirow{2}{*}{33.3} & \multirow{2}{*}{$\leq 3$} & High & $\begin{array}{l}0.25- \\
3.0\end{array}$ & 500 & $20-50$ & $30-90$ & $5-10$ & \multirow{2}{*}{14} & \multirow{2}{*}{24.5} & \multirow{2}{*}{18.4} & \multirow{2}{*}{31} \\
\hline & & & & Low & $\begin{array}{l}0.25- \\
2.75\end{array}$ & 130 & $1-2$ & 30 & $60-180$ & & & & \\
\hline \multirow{2}{*}{ Amar et al., 1997 [22] } & \multirow{2}{*}{17} & \multirow{2}{*}{38.8} & \multirow{2}{*}{2.6} & High & $\leq 3.5$ & 500 & 30 & 30 & 5 & \multirow{2}{*}{12} & \multirow{2}{*}{71} & \multirow{2}{*}{65} & \multirow{2}{*}{57} \\
\hline & & & & Low & $\leq 3.5$ & 130 & 1 & 30 & 180 & & & & \\
\hline \multirow{2}{*}{$\begin{array}{l}\text { Handforth et al., } 1998 \\
(E 05)[21]\end{array}$} & \multirow{2}{*}{196} & \multirow{2}{*}{33.1} & \multirow{2}{*}{$1-3$} & High & $<3.5$ & 500 & 30 & 30 & 5 & \multirow{2}{*}{$12-16$} & \multirow{2}{*}{27.9} & \multirow{2}{*}{12.7} & \multirow{2}{*}{23.4} \\
\hline & & & & Low & $<3.5$ & 130 & 1 & 30 & 180 & & & & \\
\hline
\end{tabular}

\section{TABLE 1: VNS Demographics and Outcomes}

AED: antiepileptic drugs, BEP: blinded evaluation period, \%SFR: percent seizure frequency reduction, SFRR: seizure frequency risk reduction, \%Resp: percent responders.

${ }^{*}$ High stimulation group results.

In the two largest trials, 352 adverse events were reported among 149 treatment group participants. The most common adverse events were current dependent and transient (occurring only during stimulus delivery), which included hoarseness, cough, and pharyngitis/throat pain accounting for 55.7\% (196/352) of all reported events $[15,21]$. Only one patient was found to withdraw from treatment as a result of an unspecified adverse event(s). Infections were only reported in the EO5 trial and occurred in $11.6 \%$ (23/198) of the patients [21]. However, a 2012 clinical practice guideline update endorsed by the American Epilepsy Society reported infections in only 3-7\% patients [23]. In general, VNS is a low-risk procedure with minor and transient adverse post-surgical events.

Deep Brain Stimulation

In 1972, seizure frequency reduction was first reported with the direct implantation of stimulators in the cerebellum [24]. The mechanism of action of DBS-ANT in epilepsy is still being investigated, but authors have suggested that the reduced seizure activity with the stimulation of the anterior nuclei of the thalamus could possibly be related to its diffuse projections to the temporal lobes and its participation in the circuit of Papez [25-26] (Figure 3). 


\section{Cureus}

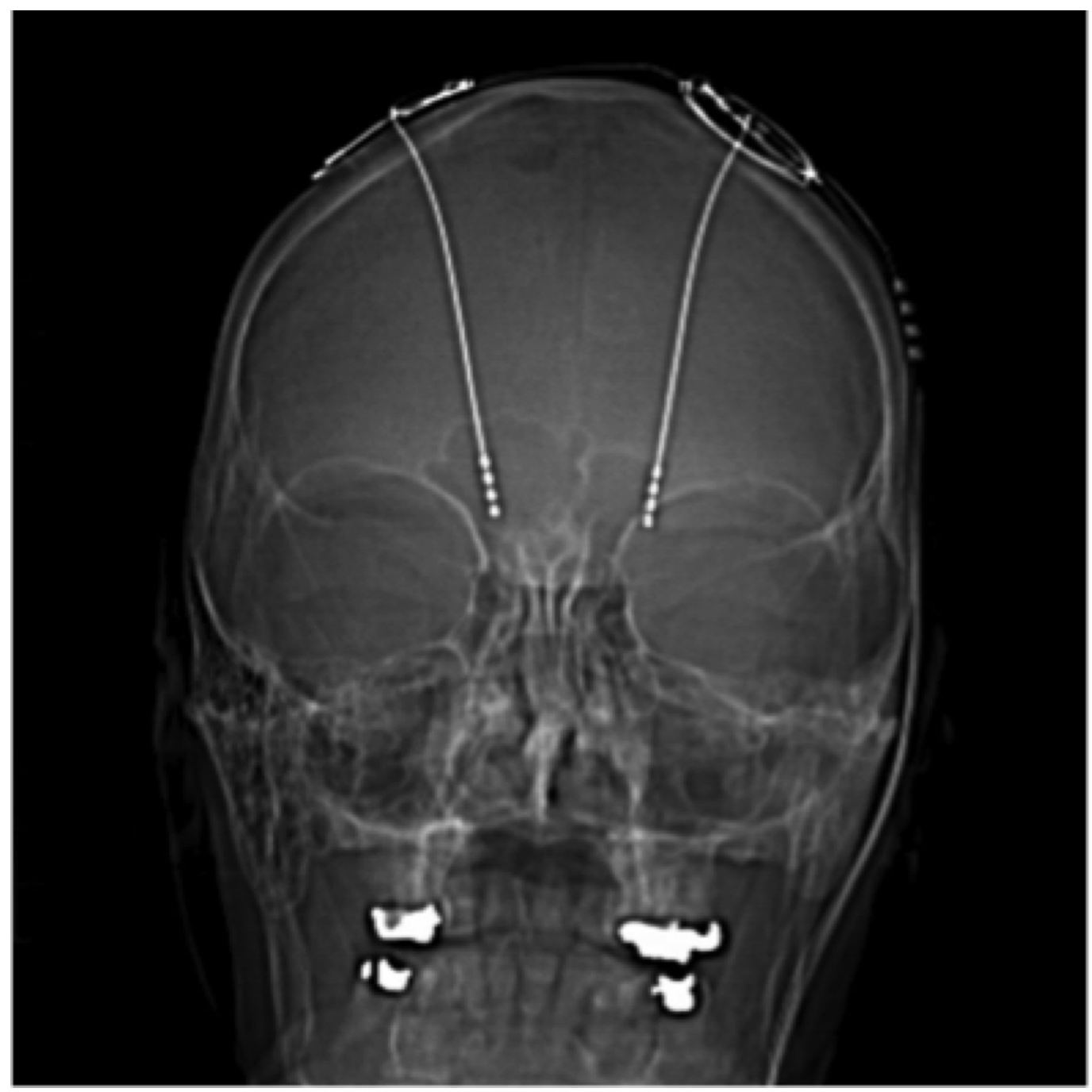

\section{FIGURE 3: Deep Brain Stimulation}

Case courtesy of A.Prof Frank Gaillard; Radiopaedia.org, rID: 2743

Recently, the double-blinded Stimulation of the Anterior Nucleus of the Thalamus for Epilepsy (SANTE) trial showed a significant decrease in seizure frequency for the treatment of DRE as shown in Table 2 [26]. However, the seizure reduction effect was found to be only significant in patients whose seizures originated from one or both temporal lobes but not in patients with frontal, parietal, occipital, or multifocal/diffuse seizure foci. In addition, the number of responders during the blinded evaluation period (BEP) was not significantly different between the groups [26]. In the 25-month, unblinded followup period, there was a statistically significant seizure frequency reduction from the baseline of $56 \%$ and a responder rate of 54\% [26]. This suggests that longer-term treatment could improve outcomes to DBSANT in patients with DRE. 


\section{Cureus}

\begin{tabular}{|c|c|c|c|c|c|c|c|c|c|c|c|c|c|}
\hline Study & $\begin{array}{l}\# \\
\text { Pts }\end{array}$ & $\begin{array}{l}\text { Age } \\
\text { (yrs) }\end{array}$ & \#AEDs & $\begin{array}{l}\text { Stimulation } \\
\text { Group }\end{array}$ & $\begin{array}{l}\text { Voltage } \\
\text { (V) }\end{array}$ & $\begin{array}{l}\text { Pulse } \\
\text { Time } \\
\text { ( } \mu \mathrm{sec} \text { ) }\end{array}$ & $\begin{array}{l}\text { Freq } \\
(\mathrm{Hz})\end{array}$ & (min) & $\begin{array}{l}\text { OFF Time } \\
(\mathrm{min})\end{array}$ & $\begin{array}{l}\text { Duration } \\
\text { of BEP } \\
\text { (weeks) }\end{array}$ & $\begin{array}{l}\% \\
\text { SFR* }\end{array}$ & SFRR ${ }^{\star}$ & $\begin{array}{l}\% \\
\text { Resp* }\end{array}$ \\
\hline \multicolumn{14}{|l|}{ DBS-ANT } \\
\hline \multirow{2}{*}{$\begin{array}{l}\text { Fisher et al., } \\
2010 \text { [26] }\end{array}$} & \multirow{2}{*}{110} & \multirow{2}{*}{36.1} & \multirow{2}{*}{$1-4$} & Active & 5 & 90 & 145 & 1 & 5 & \multirow{2}{*}{12} & \multirow{2}{*}{40.4} & \multirow{2}{*}{25.9} & \multirow{2}{*}{$29.6^{\wedge}$} \\
\hline & & & & Sham & 0 & \multicolumn{4}{|c|}{ Not set in control } & & & & \\
\hline
\end{tabular}

\section{TABLE 2: DBS-ANT Demographics and Outcomes}

AEDs: antiepileptic drugs, BEP: blinded evaluation period, \%SFR: percent seizure frequency reduction, SFRR: seizure frequency risk reduction, \%Resp: percent responders.

${ }^{*}$ Active stimulation group results.

${ }^{\wedge}$ Difference between groups were not significant.

Eight hundred and eight adverse events were reported among 109 participants following surgical implantation [26]. Device-related events occurred in 29.5\% (238/808); 6.8\% (55/808) were considered serious as most required hospitalization [26]. The most common device-related events were paresthesias (18.2\%), pain at implantation site (10.9\%), and infection (9.1\%) [26]. Five participants had hemorrhages that were neither symptomatic nor clinically significant and were detected incidentally. Six (5.6\%) participants required complete removal of the device secondary to infection, and 18 participants (16.7\%) withdrew from the trial as a result of adverse events after surgery [26].

Another potential DBS target for reducing epileptic activity is the hippocampus, also a part of the circuit of Papez. Currently, the large Controlled Randomized Stimulation Versus Resection (CoRaStir) trial is underway to assess if hippocampal stimulation will produce a greater reduction in mean monthly seizure frequency when compared to medial temporal lobe resection [27]. At the time of this review, demographics and stimulation parameters were not available to report.

Currently, DBS-ANT has been approved for use in Europe, but the FDA has not approved it for use in the United States. Should the FDA approve either DBS-ANT/hippocampal stimulation, this may provide a method for the preservation of cognitive function by allowing bilateral temporal treatment compared to patients otherwise requiring lobectomy.

\section{Responsive Cortical Neurostimulation}

In 2013, the Neuropace ${ }^{\circledR}$ RNS ${ }^{\circledR}$ System (Neuropace, Mountain View, CA) was approved by the FDA (Figure 4). Unlike VNS, which is an open loop system that delivers a preprogrammed stimulus, the RNS system is a closed-loop system and is able to detect abnormal epileptiform activity and deliver an appropriate stimulus to prevent seizure onset [28]. Permanent subdural or depth electrodes are implanted at the seizure focus area and connected to a stimulation device embedded under the scalp. The mechanism by which RNS leads to seizure frequency reduction remains unknown, but multiple mechanisms of action are hypothesized. Acute effects are thought to include the release of neurotransmitters, local cellular inhibition/excitation, as well as cerebral blood flow changes [29]. The long-term therapeutic effects may be the result of changes in neural networks secondary to synaptic plasticity, neurogenesis/cortical reorganization [29]. 


\section{Cureus}

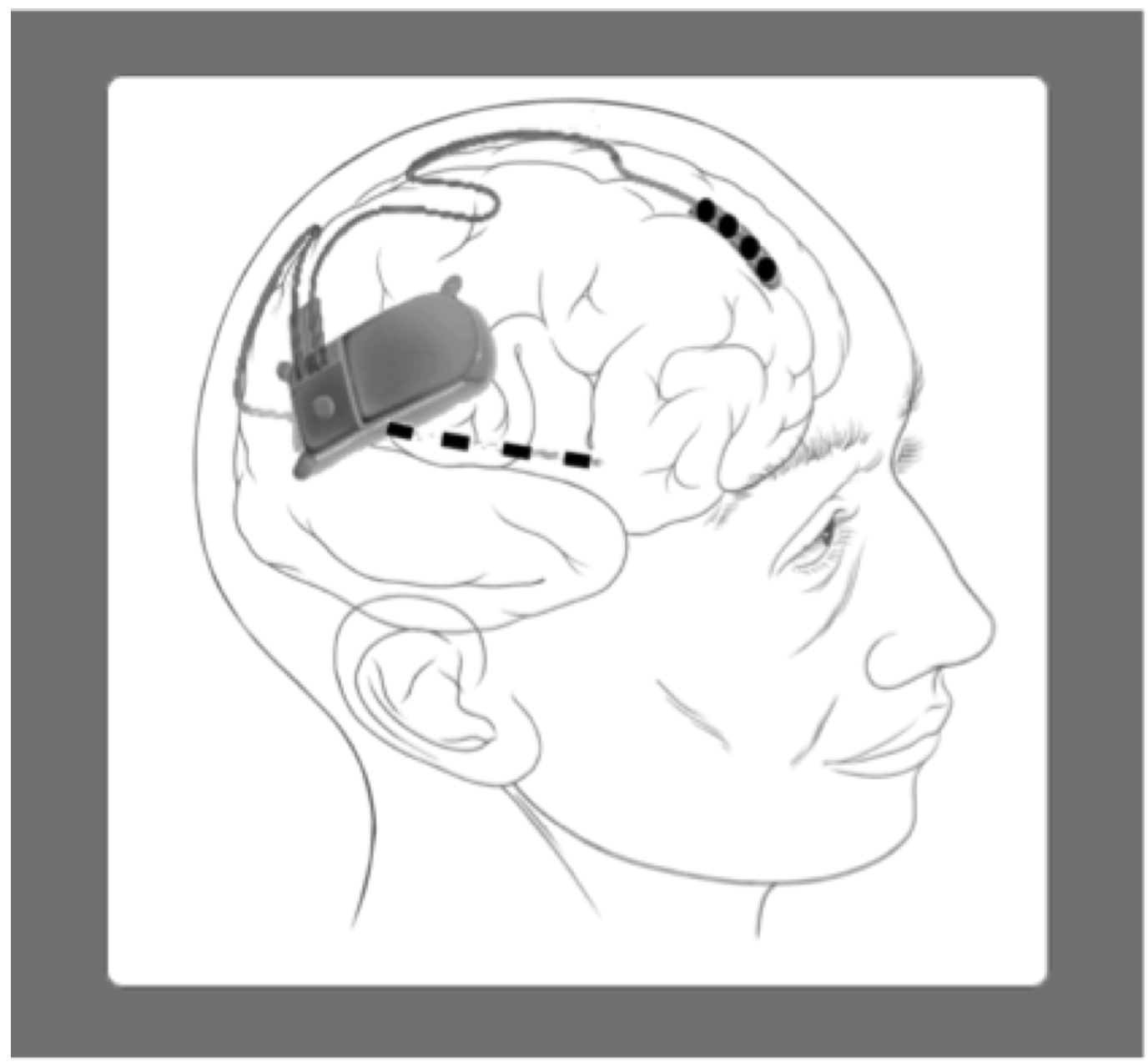

FIGURE 4: Responsive Neurostimulation

The double-blinded trial data results were published separately with the initial results including the intent-to-treat analysis and first-year, open-label period (OLP) data in 2011 [30]. This was followed by a per-protocol analysis and final clinical results in 2014 [29]. Patient demographics, stimulation parameters, and seizure frequency reduction results are summarized in Table 3. There was a significant difference in seizure frequency reduction--independent of seizure onset location--between groups. There was no significant difference demonstrated in the number of responders during the initial, blinded period. However, during the OLP the median percent reduction (53\%) and responder rate (55\%) at two years were significantly different. Again, similar to DBS-ANT, this suggests improved efficacy with an increased duration of treatment. 


\section{Cureus}

\begin{tabular}{|c|c|c|c|c|c|c|c|c|c|c|c|c|c|}
\hline Study & $\begin{array}{l}\# \\
\text { Pts }\end{array}$ & $\begin{array}{l}\text { Age } \\
\text { (yrs) }\end{array}$ & \#AEDs & $\begin{array}{l}\text { Stimulation } \\
\text { Group }\end{array}$ & $\begin{array}{l}\text { Current } \\
\text { (mA) }\end{array}$ & $\begin{array}{l}\text { Pulse } \\
\text { Time } \\
\text { ( } \mu \text { sec) }\end{array}$ & $\begin{array}{l}\text { Freq } \\
(\mathrm{Hz})\end{array}$ & $\begin{array}{l}\text { ON Time } \\
\text { (min) }\end{array}$ & $\begin{array}{l}\text { OFF } \\
\text { Time } \\
\text { (min) }\end{array}$ & $\begin{array}{l}\text { Duration of } \\
\text { BEP (weeks) }\end{array}$ & $\begin{array}{l}\% \\
\text { SFR* }\end{array}$ & SFRR* & $\begin{array}{l}\% \\
\text { Resp* }\end{array}$ \\
\hline \multicolumn{14}{|l|}{ RNS } \\
\hline Morrell et al., & \multirow{4}{*}{191} & \multirow{4}{*}{34.9} & \multirow{4}{*}{2.8} & Active & $0.5^{\star *}$ & 160 & 200 & 5.9 & - & \multirow{3}{*}{12} & \multirow{2}{*}{37.9} & \multirow{2}{*}{20.6} & \multirow{2}{*}{$29^{\wedge}$} \\
\hline & & & & Sham & Off & \multicolumn{4}{|c|}{------Not set in control group---- } & & & & \\
\hline \multirow{2}{*}{$\begin{array}{l}\text { Heck et al., } \\
2014 \text { [29] }\end{array}$} & & & & Active & $0.5^{\star \star}$ & 160 & 200 & 5.9 & - & & \multirow{2}{*}{41.5} & \multirow{2}{*}{32.1} & \multirow{2}{*}{-} \\
\hline & & & & Sham & Off & \multicolumn{4}{|c|}{--Not set in control group---- } & 12 & & & \\
\hline
\end{tabular}

\section{TABLE 3: RNS Demographics and Outcomes}

AED: antiepileptic drugs, BEP: blinded evaluation period, \%SFR: percent seizure frequency reduction, SFRR: seizure frequency risk reduction, \%Resp: percent responders.

${ }^{*}$ Active stimulation group results.

**Titrated upward as tolerated to $12 \mathrm{~mA}$.

${ }^{\wedge}$ Difference between groups not significant.

There were 206 adverse events reported among 191 participants, with the most common being implant site pain (15.7\%) and headaches (10.5\%) [30]. Five participants experienced lead damage, four of which were due to complications with lead securement at the burr hole; the other was the result of inadvertent cutting and damage to a separate strip lead that was between the skull and the titanium plate.

Postoperative intracranial hemorrhage occurred in 3.1\% (6/191), and four of these patients required hematoma evacuation [29-30]. Postoperative, device-related implant site infections were reported in seven participants (3.7\%), requiring device explantation in four patients [29-30]. The reported infections were of soft-tissue only and did not involve the brain or cranium [29-30]. Five participants withdrew from the study, none of which were the result of adverse events [29-30].

\section{Transcranial Direct Current Stimulation}

tDCS is a non-invasive application of direct current to the scalp that subsequently causes stimulation of the cerebral cortex (Figure 5). The exact mechanism of tDCS in DRE is not completely known, but it is thought to be related to the hyperpolarization and depolarization of neurons that cause cortical inhibition at the epileptic foci with an anode-to-cathode direction of current [31]. Pharmacologic and magnetic resonance spectroscopy studies have demonstrated that the mechanism for tDCS could be the result of local neuronal effects secondary to NMDA receptor modulation, and reductions in glutamate and GABA activity [32-33]. 


\section{Cureus}

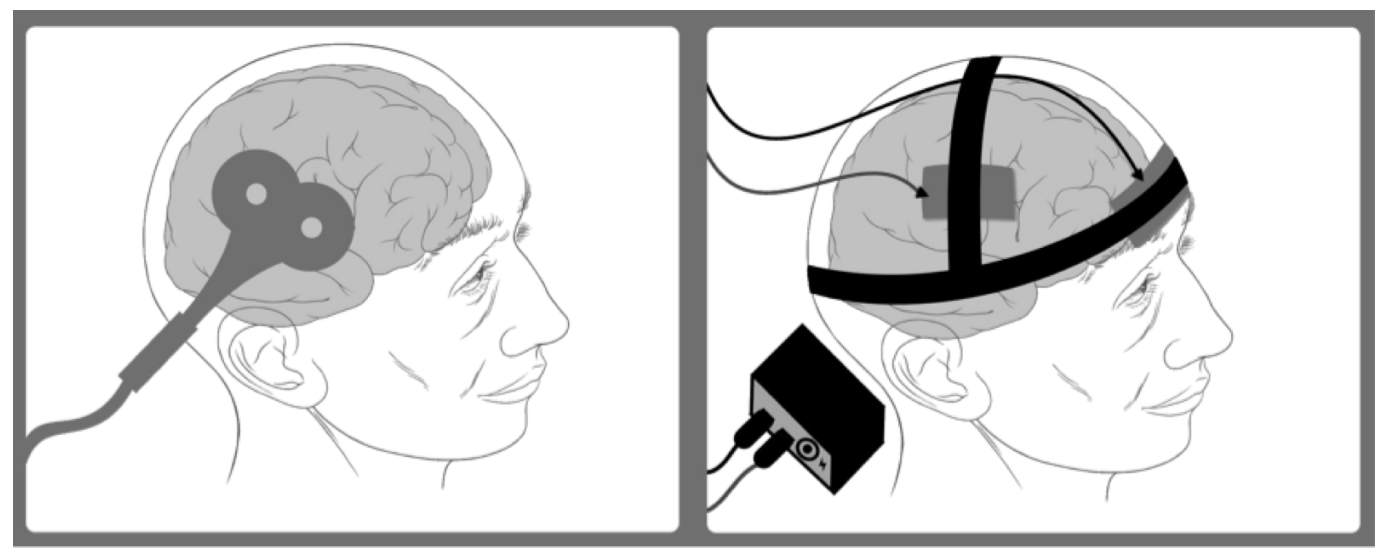

FIGURE 5: rTMS Device (Left), tDCS Device (Right)

There has been only one double-blinded RCT published; the results of which are summarized in Table 4 [34]. No statistically significant reductions in seizure frequency were found, but the treatment group showed a reduction in epileptiform discharge up to four weeks post-treatment.

\begin{tabular}{|c|c|c|c|c|c|c|c|c|c|c|c|}
\hline Study & $\begin{array}{l}\# \\
\text { Pts }\end{array}$ & $\begin{array}{l}\text { Age } \\
\text { (yrs) }\end{array}$ & \#AEDs & $\begin{array}{l}\text { Stimulus } \\
\text { Location }\end{array}$ & $\begin{array}{l}\text { Stimulation } \\
\text { Group }\end{array}$ & $\begin{array}{l}\text { Current } \\
\text { (mA) }\end{array}$ & $\begin{array}{l}\text { ON } \\
\text { Time }\end{array}$ & $\begin{array}{l}\text { Duration } \\
\text { of BEP }\end{array}$ & $\begin{array}{l}\% \\
\text { SFR* }\end{array}$ & SFRR* & $\begin{array}{l}\% \\
\text { Resp }\end{array}$ \\
\hline \multicolumn{12}{|l|}{ tDCS } \\
\hline \multirow{2}{*}{$\begin{array}{l}\text { Fregni et al., } 2006 \\
\text { [34] }\end{array}$} & \multirow{2}{*}{19} & \multirow{2}{*}{24.2} & \multirow{2}{*}{2.3} & \multirow{2}{*}{ Seizure Focus } & Active & 1 & $20 \mathrm{~min}$ & \multirow{2}{*}{30 days } & \multirow{2}{*}{$44^{\wedge}$} & \multirow{2}{*}{32.9} & \multirow{2}{*}{ - } \\
\hline & & & & & Sham & 1 & $30 \mathrm{sec}$ & & & & \\
\hline
\end{tabular}

\section{TABLE 4: tDCS Demographics and Outcomes}

AED: antiepileptic drugs, BEP: blinded evaluation period, \%SFR: percent seizure frequency reduction, SFRR: seizure frequency risk reduction, \%Resp: percent responders.

${ }^{*}$ Active stimulation group results.

${ }^{\wedge}$ Difference between groups not significant.

Adverse events with tDCS are minimal as a limited number of minor adverse events reported at the site of stimulation; four patients (21.1\%) reported mild itching [34].

Currently, tDCS is not FDA approved for clinical use. The current data for tDCS may support the idea that it may be safe, but is insufficient to make any clinically relevant conclusions regarding its therapeutic use for DRE, and further studies are needed.

\section{Transcranial Magnetic Stimulation}

rTMS is a repetitive, electrical, non-invasive brain stimulation (NIBS) method that has been shown to be effective in some psychiatric and neurologic disorders (Figure 5). In rTMS, an alternating current is run through a wire coil to create a fluctuating magnetic field that is then focused on the area of interest in the brain, inducing intracranial electrical currents [35]. Excitation of the cortex is enhanced by highfrequency stimulation $(>1 \mathrm{~Hz})$, while low stimulation rates $(\leqslant 1 \mathrm{~Hz})$ reduce cortical excitation [36-37]. Different coils are designed to stimulate either superficial and smaller foci or deeper and broader foci [38]. 


\section{Cureus}

The exact mechanism of action is still unknown. It is thought to be related to the currents generated by the magnetic field, which lead to changes in the concentration of neurotransmitters (primarily glutamate and dopamine), and has been shown to be related to stimulation intensity and frequency delivered [36, 39-40]. Clinical effects of rTMS are related to the temporal spacing and duration of the stimulation, and it is believed that induced cortical plasticity may be the result of NMDA receptor modulation [41-42].

The use of rTMS as a non-invasive, antiepileptic therapeutic option for patients with DRE has had mixed results based on the four published, randomized controlled trials summarized in Table 5. In two trials-Theodore et al., and Cantello et al.,--no significant differences in seizure frequency reduction were found [43-44]. In contrast, two studies have demonstrated significant reductions in seizure frequency in patients with a predetermined location of cortical seizure foci $[41,45]$. Both of these studies included patients with cortical dysplasia or superficial epilepsy origin, which had been excluded in previous trials [43]. A significant decrease in seizure frequency, eight weeks after stimulation, was demonstrated suggesting that the initial rTMS treatment had a longer-lasting effect on seizure frequency reduction, even after the scheduled treatment was completed. Additionally, Sun et al., (a single-blinded trial) demonstrated that $35.5 \%$ of patients remained seizure free, and $22.6 \%$ of patients had a complete abolishment of epileptiform discharges at the end of the blinded evaluation period (BEP).

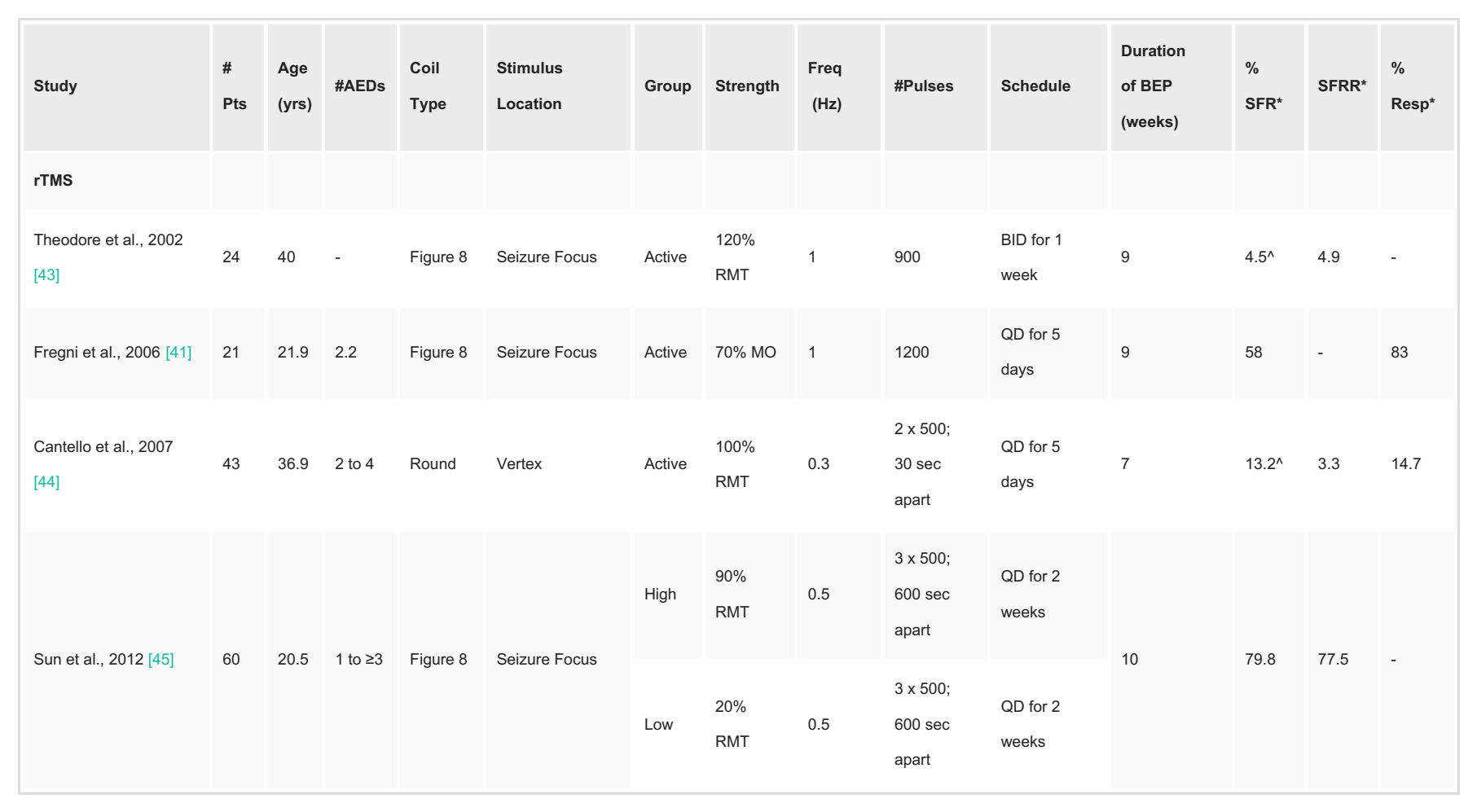

\section{TABLE 5: rTMS Demographics and Outcomes}

AED: antiepileptic drugs, BEP: blinded evaluation period, \%SFR: percent seizure frequency reduction, SFRR: seizure frequency risk reduction, \%Resp: percent responders, MO: maximum output, RMT: resting motor threshold, BID: twice daily, QD: daily.

${ }^{*}$ Active stimulation group results or High Stimulation Group.

^Difference between groups not significant.

Other authors have noted that the reason for the non-significant results for Theodore et al., may have been secondary to their recruitment of subjects with deep epileptic origin [39]. This results in a decreased applied magnetic field whose strength is a cubic function of the distance between the rTMS device and the seizure foci being stimulated [43]. A greater, but still non-significant, improvement was observed among study participants who had a more lateral temporal seizure foci compared to those with deeper mesial 
temporal foci $[39,43]$.

The Cantello, et al. trial randomized patients to treatment with stimulation from a circular coil, based on previous reports of efficacy [44]. The authors attributed their poor results to the unpredictable variability in seizure frequency in relation to their sample size [44]. However, round coils have been shown to provide a more diffuse and deep effect compared to the focused and superficial effects seen with figureeight coils [38]. For successful application of rTMS in the treatment of DRE, stimulation at the exact location of the seizure focus is critical [41]. This explanation offers a better reason for the lack of significant seizure reduction difference between groups in this particular study.

Among all rTMS trials reviewed, the vast majority of adverse events were minor, occurring in 19 (12.8\%) of the collective 148 participants [41, 43-45]. The most commonly reported events included headache, dizziness, and tinnitus. One patient on two occasions experienced a typical complex partial seizure, and as a result did not complete the entire week of stimulation but remained a part of the trial [43]. Patients should be cautioned that rTMS could induce seizures in those who are already seizure-prone versus nonepileptic individuals. However, a literature review of rTMS in 280 DRE patients found only four patients who had a seizure during treatment, only one of whom had an atypical seizure whose focus was different from their previously noted seizure origin. Importantly, this event occurred in a participant who was receiving a high-frequency $(16 \mathrm{~Hz})$ stimulation, which has been shown to cause cortical excitation [46]. The risk of additional seizures with rTMS stimulation in patients with DRE was nearly as safe to those without epilepsy [46].

\section{Primary outcome reporting}

All studies included in this review reported mean/median seizure frequency reduction as the primary outcome and measure of clinical improvement. However, in some trials no significant differences were found in the reported number of percent responders, those participants with a $\geqslant 50 \%$ seizure frequency reduction, suggesting that a majority of patients who benefited during this time may have had only modest results [26, 30]. A reasonable alternative might be to report percent responders as the primary outcome. This would correlate better to consecutive seizure-free days in patients. Additionally, reporting of seizure frequency risk reduction (SFRR)--synonymous with absolute risk reduction, calculated as the difference in seizure frequency between both groups--would allow reporting of the number needed to treat (NNT). The same method could be applied to percent responders. The authors suggest that increasing the reporting of SFRR and percent responder data would improve the quality of clinically relevant outcome data available.

\section{Limitations of non-invasive stimulation studies}

Sham Protocols

The design of nervous stimulation trials, both IBS and NIBS, can be complicated by the difficulty of reproducing sham conditions that replicate active treatment and maintain participant blinding in order to minimize placebo effect. A reliable sham protocol for tDCS has been described as giving a short (30 sec) stimulus, which gives the initial sensation of treatment but maintains blinding because of the fast attenuation of the stimulus in those with a sustained stimulation [47]. Sham is a much greater concern in rTMS trials because the stimulation is accompanied by a loud sound, with strong scalp sensations and muscle twitching. Thus, patients can become unblinded during titration of the resting membrane threshold (RMT). Fregni, et al. based their stimulation rTMS protocol on the maximal output of the device and the use of a replica that produced a similar sound artifact, which required no titration to RMT avoiding this limitation [41]. Other rTMS studies have utilized sham procedures that included rotation or increased distance of the device from the scalp [43-44]. Sun et al., used a low-intensity RMT stimulus protocol for sham, and neither group received 100\% RMT. This suggests blinding may have been preserved. However, there was no discussion of the differences, if any, in sensations among the groups. The use of focal electrical stimulation and personalized titration to simulate rTMS stimulation has also been investigated and described in a small study as a possible alternative sham protocol, but further validation is needed [48]. Nonetheless, a measure of the sham effect for three out of the four rTMS trials in this review was shown to have a minimal effect on seizure frequency reduction and responder rates [49]. The use of "active control groups" (in VNS and rTMS) differs from truly blinded patients, and this difference in data quality can affect the comparison of IBS and NIBS protocols. Future RCTs for nervous stimulation should include participants who are naïve to therapy, crossover study designs should be 


\section{Cureus}

avoided, and care should be taken to ensure participants receive therapy separately from other study participants.

\section{Stimulation Variability}

The standards for stimulation parameters between brain stimulation trials are very heterogeneous with respect to applied current, frequency, strength, duration, and the number of treatment sessions. Ziemann, et al. discussed the further complicating issue of inter-subject and inter-session variability of plasticity induction among NIBS studies and suggest that researchers design experimental and stimulationpriming protocols that are carefully standardized by collecting EEG recordings during stimulation [50]. This would allow for the continued investigation and determination of the underlying causes of intersession and inter-subject variability in order to develop techniques to limit such variability and improve clinical efficacy.

\section{Conclusions}

Despite improvements, the localization of surgically amendable seizure foci remains difficult due to diffuse epileptogenic foci and the presence of encephalomalacia in post-traumatic DRE (PTDRE) patients. For affected service members, this makes identifying alternative therapies that are efficacious a worthy undertaking. To the authors' knowledge, no such studies have been conducted specifically investigating the efficacy of nervous stimulation in the PTDRE population. As described in detail above and summarized in Table 6, the evidence for the efficacy of NIBS for DRE in the general population has been growing, but primary outcome results are still far less convincing than that seen with IBS or surgical resection in eligible patients. The use of tDCS and rTMS for the treatment of DRE are still investigational, and there are currently no FDA-approved indications for their use. The IBS therapies for the treatment of DRE have been shown in multiple, large, blinded, randomized controlled trials to be effective options for reducing seizure frequency and are currently in clinical use. The authors' hope is that this review encourages future funding for research investigating the role of NIBS and IBS therapies for PTDRE. Research and treatments should focus on improving data reporting, improving sham protocols, and reducing stimulation response variability in order to determine practical and clinically effective alternative treatment options where surgery is either not an option, has failed, or for patients who prefer a non-invasive approach. 


\section{Cureus}

\begin{tabular}{|c|c|c|c|c|}
\hline Study & FDA Approved & $\%$ SFR* & SFRR ${ }^{*}$ & $\% \operatorname{Resp}^{*}$ \\
\hline VNSSG 1995 (E03) [15] & Y & 24.5 & 18.4 & 31 \\
\hline Amar et al., 1997 [22] & Y & 71 & 65 & 57 \\
\hline Handforth et al., 1998 (E05) [21] & $\mathrm{Y}$ & 27.9 & 12.7 & 23.4 \\
\hline Fisher et al., 2010 (SANTE) [26] & $\mathrm{N}$ & 40.4 & 25.9 & $29.6^{\wedge}$ \\
\hline Morrell et al., 2011 [30] & Y & $3 / .9$ & 20.6 & $29^{\wedge}$ \\
\hline Heck et al., 2014 [29] & Y & 41.5 & 32.1 & - \\
\hline Fregni et al., 2006 [34] & $\mathrm{N}$ & 44 & 32.9 & - \\
\hline Theodore et al., 2002 [43] & $\mathrm{N}$ & $4.5^{\wedge}$ & 4.9 & - \\
\hline Fregni et al., 2006 [41] & $\mathrm{N}$ & 58 & - & 83 \\
\hline Cantello et al., 2007 [44] & $\mathrm{N}$ & $13.2^{\wedge}$ & 3.3 & 14.7 \\
\hline Sun et al., 2012 [45] & $\mathrm{N}$ & 79.8 & 77.5 & - \\
\hline
\end{tabular}

\section{TABLE 6: Summary}

\%SFR: percent seizure frequency reduction, SFRR: seizure frequency risk reduction, \%Resp: percent responders.

${ }^{*}$ Active stimulation group results.

${ }^{\wedge}$ Difference between groups not significant.

\section{Additional Information Disclosures}

Conflicts of interest: In compliance with the ICMJE uniform disclosure form, all authors declare the following: Payment/services info: All authors have declared that no financial support was received from any organization for the submitted work. Financial relationships: All authors have declared that they have no financial relationships at present or within the previous three years with any organizations that might have an interest in the submitted work. Other relationships: All authors have declared that there are no other relationships or activities that could appear to have influenced the submitted work.

\section{References}

1. Committee on the Public Health Dimensions of the Epilepsies, Board on Health Sciences Policy, Institute of Medicine of the National Academies: Epilepsy Across the Spectrum: Promoting Health and Understanding. National Academy of Sciences. England MJ, Liverman CT, Schultz AM, Strawbridge LM (ed): National Academies Press, Washington DC; 2012. 20.

2. Annegers JF, Hauser WA, Coan SP, Rocca WA: A population-based study of seizures after traumatic brain injuries. N Engl J Med. 1998, 338:20-24. 10.1056/NEJM199801013380104

3. Diaz-Arrastia R, Agostini MA, Madden CJ, Van Ness PC: Posttraumatic epilepsy: the endophenotypes of a human model of epileptogenesis. Epilepsia. 2009, 50 Suppl 2:14-20. 10.1111/j.1528-1167.2008.02006.x

4. Lowenstein DH: Epilepsy after head injury: an overview . Epilepsia. 2009, 50 Suppl 2:4-9. 10.1111/j.15281167.2008.02004.x

5. Irimia A, Van Horn JD: Epileptogenic focus localization in treatment-resistant post-traumatic epilepsy . J Clin Neurosci. 2015, 22:627-631. 10.1016/j.jocn.2014.09.019

6. Dudek FE, Sutula TP: Epileptogenesis in the dentate gyrus: a critical perspective. Prog Brain Res. 2007, 163:755-773. 10.1016/S0079-6123(07)63041-6

7. Haltiner AM, Temkin NR, Dikmen SS: Risk of seizure recurrence after the first late posttraumatic seizure . 
Arch Phys Med Rehabil. 1997, 78:835-840. 10.1016/S0003-9993(97)90196-9

8. Hess CP, Barkovich AJ: Seizures: emergency neuroimaging. Neuroimaging Clin N Am. 2010, 20:619-637. 10.1016/j.nic.2010.07.013

9. Brain Trauma Foundation, American Association of Neurological Surgeons, Congress of Neurological Surgeons, et al.: XIII. Antiseizure prophylaxis. J Neurotrauma. 2007, 24 Supp 1:S83-S86. 10.1089/neu.2007.9983

10. Kwan P, Schachter SC, Brodie MJ: Drug-resistant epilepsy. N Engl J Med. 2011, 365:919-926. 10.1056/NEJMra1004418

11. Semah F, Picot MC, Adam C, et al.: Is the underlying cause of epilepsy a major prognostic factor for recurrence?. Neurology. 1998, 51:1256-1262. 10.1212/WNL.51.5.1256

12. Litt B, Echauz J: Prediction of epileptic seizures . Lancet Neurol. 2002, 1:22-30. 10.1016/s14744422(02)00003-0

13. Schuele SU, Lüders HO: Intractable epilepsy: management and therapeutic alternatives . Lancet Neurol. 2008, 7:514-524. 10.1016/S1474-4422(08)70108-X

14. Medical Devices, VNS Therapy System - P970003s050 (2013). Accessed: October 13, 2014: http://www.fda.gov/MedicalDevices/ProductsandMedicalProcedures/DeviceApprovalsandClearances/Recen ApprovedDevices/....

15. The Vagus Nerve Stimulation Study Group: A randomized controlled trial of chronic vagus nerve stimulation for treatment of medically intractable seizures. Neurology. 1995, 45:224-230. 10.1212/WNL.45.2.224

16. Rutecki P: Anatomical physiological, and theoretical basis for the antiepileptic effect of vagus nerve stimulation. Epilepsia. 1990, 31 Suppl 2:S1-S6. 10.1111/j.1528-1157.1990.tb05843.x

17. McLachlan RS: Suppression of interictal spikes and seizures by stimulation of the vagus nerve . Epilepsia. 1993, 34:918-923. 10.1111/j.1528-1157.1993.tb02112.x

18. Krahl SE, Clark KB, Smith DC, Browning RA: Locus coeruleus lesions suppress the seizure-attenuating effects of vagus nerve stimulation. Epilepsia. 1998, 39:709-714. 10.1111/j.1528-1157.1998.tb01155.x

19. Ben-Menachem E, Hamberger A, Hedner T, et al.: Effects of vagus nerve stimulation on amino acids and other metabolites in the CSF of patients with partial seizures. Epilepsy Res. 1995, 20:221-227. 10.1016/0920-1211(94)00083-9

20. Henry TR: Therapeutic mechanisms of vagus nerve stimulation. Neurology. 2002, 59:S3-S14. 10.1212/WNL.59.6_suppl_4.S3

21. Handforth A, DeGiorgio CM, Schachter SC, et al.: Vagus nerve stimulation therapy for partial-onset seizures: a randomized active-control trial. Neurology. 1998, 51:48-55. 10.1212/WNL.51.1.48

22. Amar AP, Heck CN, Levy ML, et al.: An institutional experience with cervical vagus nerve trunk stimulation for medically refractory epilepsy: rationale, technique, and outcome. Neurosurgery. 1998, 43:1265-1276.

23. Morris GL 3rd, Gloss D, Buchhalter J, Mack KJ, Nickels K, Harden C: Evidence-based guideline update: vagus nerve stimulation for the treatment of epilepsy: report of the guideline development subcommittee of the american academy of neurology. Epilepsy Curr. 2013, 13:297-303. 10.5698/15357597-13.6.297

24. Cooper IS, Amin I, Gilman S: The effect of chronic cerebellar stimulation upon epilepsy in man . Trans Am Neurol Assoc. 1973, 98:192-196.

25. Fisher RS: Deep brain stimulation for epilepsy. Handb Clin Neurol. 2013, 116:217-234. 10.1016/B978-0444-53497-2.00017-6

26. Fisher R, Salanova V, Witt T, et al.: Electrical stimulation of the anterior nucleus of thalamus for treatment of refractory epilepsy. Epilepsia. 2010, 51:899-908. 10.1111/j.1528-1167.2010.02536.x

27. Controlled Randomized Stimulation Versus Resection (CoRaStiR) . (2014). Accessed: December 10, 2014: http://clinicaltrials.gov/ct2/show/NCT00431457.

28. Morrell M: Brain stimulation for epilepsy: can scheduled or responsive neurostimulation stop seizures? Curr Opin Neurol. 2006, 19:164-168. 10.1097/01.wco.0000218233.60217.84

29. Heck CN, King-Stephens D, Massey AD, et al.: Two-year seizure reduction in adults with medically intractable partial onset epilepsy treated with responsive neurostimulation: final results of the RNS System Pivotal trial. Epilepsia. 2014, 55:432-441. 10.1111/epi.12534

30. Morrell MJ, RNS System in Epilepsy Study Group: Responsive cortical stimulation for the treatment of medically intractable partial epilepsy. Neurology. 2011, 77:1295-1304. 10.1212/WNL.0b013e3182302056

31. Kabakov AY, Muller PA, Pascual-Leone A, Jensen FE, Rotenberg A: Contribution of axonal orientation to pathway-dependent modulation of excitatory transmission by direct current stimulation in isolated rat hippocampus. J Neurophysiol. 2012, 107:1881-1889. 10.1152/jn.00715.2011

32. Nitsche MA, Fricke K, Henschke U, et al.: Pharmacological modulation of cortical excitability shifts induced by transcranial direct current stimulation in humans. J Physiol. 2003, 553:293-301. 10.1113/jphysiol.2003.049916

33. Stagg CJ, Best JG, Stephenson MC, et al.: Polarity-sensitive modulation of cortical neurotransmitters by transcranial stimulation. J Neurosci. 2009, 29:5202-5206. 10.1523/JNEUROSCI.4432-08.2009

34. Fregni F, Thome-Souza S, Nitsche MA, Freedman SD, Valente KD, Pascual-Leone A: A controlled clinical 
trial of cathodal DC polarization in patients with refractory epilepsy. Epilepsia. 2006, 47:335-342. 10.1111/j.1528-1167.2006.00426.x

35. Hallett M: Transcranial magnetic stimulation and the human brain. Nature. 2000, 406:147-150. $10.1038 / 35018000$

36. Chen R, Classen J, Gerloff C, et al.: Depression of motor cortex excitability by low-frequency transcranial magnetic stimulation. Neurology. 1997, 48:1398-1403. 10.1212/WNL.48.5.1398

37. Maeda F, Keenan JP, Tormos JM, Topka H, Pascual-Leone A: Modulation of corticospinal excitability by repetitive transcranial magnetic stimulation. Clin Neurophysiol. 2000, 111:800-805. 10.1016/S13882457(99)00323-5

38. Rossi S, Hallett M, Rossini PM, Pascual-Leone A, Safety of TMS Consensus Group: Safety, ethical considerations, and application guidelines for the use of transcranial magnetic stimulation in clinical practice and research. Clin Neurophysiol. 2009, 120:2008-2039. 10.1016/j.clinph.2009.08.016

39. Hemond CC, Fregni F: Transcranial magnetic stimulation in neurology: what we have learned from randomized controlled studies. Neuromodulation. 2007, 10:333-344. 10.1111/j.1525-1403.2007.00120.x

40. Pascual-Leone A, Amedi A, Fregni F, Merabet LB: The plastic human brain cortex. Annu Rev Neurosci. 2005, 28:377-401. 10.1146/annurev.neuro.27.070203.144216

41. Fregni F, Otachi PT, Do Valle A, et al.: A randomized clinical trial of repetitive transcranial magnetic stimulation in patients with refractory epilepsy. Ann Neurol. 2006, 60:447-455. 10.1002/ana.20950

42. Joo EY, Han SJ, Chung SH, Cho JW, Seo DW, Hong SB: Antiepileptic effects of low-frequency repetitive transcranial magnetic stimulation by different stimulation durations and locations. Clin Neurophysiol. 2007, 118:702-708. 10.1016/j.clinph.2006.11.008

43. Theodore WH, Hunter K, Chen R, et al.: Transcranial magnetic stimulation for the treatment of seizures: A controlled study. Neurology. 2002, 59:560-562. 10.1212/WNL.59.4.560

44. Cantello R, Rossi S, Varrasi C, et al.: Slow repetitive TMS for drug-resistant epilepsy: clinical and EEG findings of a placebo-controlled trial. Epilepsia. 2007, 48:366-374. 10.1111/j.1528-1167.2006.00938.x

45. Sun W, Mao W, Meng X, Wang D, Qiao L, Tao W, et al: Low-frequency repetitive transcranial magnetic stimulation for the treatment of refractory partial epilepsy: a controlled clinical study. Epilepsia. 2012, 53:1782-1789. 10.1111/j.1528-1167.2012.03626.x

46. Bae EH, Schrader LM, Machii K, et al.: Safety and tolerability of repetitive transcranial magnetic stimulation in patients with epilepsy: a review of the literature. Epilepsy Behav. 2007, 10:521-528. 10.1016/j.yebeh.2007.03.004

47. Gandiga PC, Hummel FC, Cohen LG: Transcranial DC stimulation (tDCS): A tool for double-blind shamcontrolled clinical studies in brain stimulation. Clin Neurophysiol. 2006, 117:845-850. 10.1016/j.clinph.2005.12.003

48. Arana AB, Borckardt JJ, Ricci R, et al.: Focal electrical stimulation as a sham control for repetitive transcranial magnetic stimulation: Does it truly mimic the cutaneous sensation and pain of active prefrontal repetitive transcranial magnetic stimulation?. Brain Stimul. 2008, 1:44-51. 10.1016/j.brs.2007.08.006

49. Bae EH, Theodore WH, Fregni F, Cantello R, Pascual-Leone A, Rotenberg A: An estimate of placebo effect of repetitive transcranial magnetic stimulation in epilepsy. Epilepsy Behav. 2011, 20:355-359. 10.1016/j.yebeh.2010.12.005

50. Ziemann U, Siebner HR: Inter-subject and inter-session variability of plasticity induction by noninvasive brain stimulation: boon or bane?. Brain Stimul. 2015, 8:662-663. 10.1016/j.brs.2015.01.409 